\title{
AVALIAÇÃO DO USO DE IMIDACLOPRID NO CONTROLE DE Acromyrmex spp. (HYMENOPTERA: FORMICIDAE) EM PLANTIO DE Pinus taeda
}

\author{
Marcelo Diniz Vitorino ${ }^{1}$, Raphaela Noêmia Dutra², Taise Cristina Plattau Arenhardt², \\ Liliam Cristiane Beal ${ }^{3}$, Adam Henry Marques Gonçalves ${ }^{2}$, Rafaela Tamara Marquardt ${ }^{4}$, \\ Paula Adriana da Costa ${ }^{2}$, Sandra Ciriaco de Cristo ${ }^{4}$ \\ ${ }^{1}$ Fundação Universidade Regional de Blumenau, Departamento de Engenharia Florestal, Blumenau, Santa Catarina, Brasil - \\ dinizvitorino@gmail.com \\ ${ }^{2}$ Fundação Universidade Regional de Blumenau, Curso de Engenharia Florestal, Blumenau, Santa Catarina, Brasil - \\ raphaelandutra@gmail.com; taise.arenhardt@hotmail.com; adam_henryy@hotmail.com; paulacostaflorestal@gmail.com \\ ${ }^{3}$ Fundação Universidade Regional de Blumenau, Laboratório de Monitoramento e Proteção Florestal, Blumenau, Santa Catarina, \\ Brasil - liliambeal@hotmail.com \\ ${ }^{4}$ Fundação Universidade Regional de Blumenau, Programa de Pós-Graduação em Engenharia Florestal, Blumenau, Santa Catarina, \\ Brasil - rafaela.ef@gmail.com; sandraciriaco@hotmail.com
}

Recebido para publicação: 13/05/2013 - Aceito para publicação: 14/07/2014

\begin{abstract}
Resumo
Este trabalho teve o objetivo de avaliar a utilização do ingrediente ativo imidacloprid, aplicado em diferentes dosagens, por meio de gel condicionador de solo, em mudas de Pinus taeda L. (Pinaceae) recém-plantadas. $\mathrm{O}$ experimento foi instalado em uma das áreas da Empresa Celulose Irani S.A., no município de Vargem Bonita, SC, com desenho experimental em blocos ao acaso com 5 tratamentos e 8 repetições. As avaliações foram realizadas aos 25,56 e 81 dias após a instalação do experimento. A análise estatística mostrou não haver diferença significativa entre os tratamentos. A presença da APP próxima ao experimento influenciou significativamente nos resultados. A intensidade de dano após três avaliações (81 dias) foi de 4,06\%, resultado este promissor para o controle de quenquéns (Acromyrmex spp.) com a metodologia proposta.

Palavras-chave: Controle químico; formigas cortadeiras; avaliação de dano.
\end{abstract}

\begin{abstract}
Evaluation of imidacloprid usage in control of Acromyrmex spp. in Pinus taeda planting. This research aimed to evaluate usage of active ingredient imidacloprid in different dosages, applied as gel soil conditioner in seedlings of Pinus taeda L. (Pinaceae) newly planted. The experiment was installed in one area of the company Celulose Irani S. A., at Vargem Bonita city, Santa Catarina State. The experimental design was randomized blocks with five treatments and 8 replicates. Evaluations were performed at 25, 56 and 81 days after the experiment beginning. The presence of Permanent Preservation Areas (APP) near the experimental area significantly influenced the outcome. The intensity of damage after three assessments (81 days) was $4.06 \%$, a promising result for control leafcutting ants (Acromyrmex spp.) with the proposed methodology.

Keywords: Chemical control; leaf-cutting ants; damage assessment.
\end{abstract}

\section{INTRODUÇÃO}

Características como rápido crescimento e boa adaptação às condições edafoclimáticas fizeram das espécies Eucalyptus spp. e Pinus spp. as mais utilizadas em programas de reflorestamento no Brasil. Essas espécies, inicialmente, foram pouco atacadas por insetos-pragas quando introduzidas, principalmente por esses insetos ainda não estarem adaptados às mesmas (RIBEIRO; MENDONÇA, 2004). Entre os insetos considerados pragas em silvicultura, as formigas cortadeiras são apontadas como as mais importantes nos reflorestamentos de eucalipto e pínus, em razão dos prejuízos que causam e de sua vasta ocorrência (ANTUNES; DELLA LUCIA, 1999). No caso das mudas de Pinus taeda L., o

FLORESTA, Curitiba, PR, v. 45, n. 1, p. 41 - 48, jan. / mar. 2015.

Vitorino, M. D. et al.

ISSN eletrônico 1982-4688 / ISSN impresso 0015-3826

41

DOI: $10.5380 /$ rf.v45i1.31912 
ataque de formigas cortadeiras tem início na parte basal das plantas, podendo chegar ao ápice e até cortar o meristema apical (NICKELE, 2008), comportamento este semelhante para outras espécies do gênero Pinus.

As perdas causadas por formigas cortadeiras variam de acordo com a idade da planta e a intensidade do ataque. Em casos de ataques intensos e constantes, as perdas podem chegar a $100 \%$ da produção. Existe redução significativa no desenvolvimento de plantas atacadas por formigas cortadeiras durante os primeiros 2 anos de idade, sendo que os parâmetros afetados são o diâmetro, a altura e o índice de produtividade, e, quando o ataque ocorre na fase inicial do plantio, as perdas podem ser irreversíveis (ANJOS et al., 1993; CANTARELLI et al., 2008). Mudas que sofrem desfolha superior a $50 \%$ aos 30 dias de idade do plantio têm o crescimento em diâmetro e altura afetados, sendo que a desfolha total pode causar a mortalidade das plantas (NICKELE, 2008).

O controle à formiga cortadeira é fundamental em reflorestamentos, uma vez que elas constituem fator limitante ao seu desenvolvimento, causando perdas diretas, como a morte de mudas e a redução do crescimento de árvores, e indiretas, como a diminuição da resistência das árvores a outros insetos e agentes patogênicos.

De acordo com Mendes Filho (1981), o investimento para o controle de formigas cortadeiras pode chegar a $30 \%$ do custo da floresta ao final do $3^{\circ}$ ciclo, o que, de acordo com Rezende et al. (1983), corresponde a $7,41 \%$ do preço da madeira em pé. Vilela (1986) observou que as formigas cortadeiras representam $75 \%$ dos custos e do tempo gasto no controle de pragas.

A maioria das empresas do setor florestal brasileiro adota, para o controle das formigas cortadeiras, iscas formicidas à base dos ingredientes ativos fipronil e sulfluramida e, em grande parte delas, a quantidade de iscas aplicadas em campo não leva em consideração a infestação de formigas cortadeiras. Dessa forma, aplica-se uma quantidade de formicida que pode chegar a até 5 vezes mais do que seria recomendado caso fosse considerada a infestação (VITORINO et al., 2012; ARENHARDT et al., 2012).

A redução do impacto ambiental e questões econômicas têm levado empresas do setor florestal a buscar alternativas para melhorar as técnicas de controle químico e reduzir os efeitos dos inseticidas no ambiente. As empresas florestais que são certificadas pelo Forest Stewardship Council (FSC) têm até o ano de 2015 para reduzir o uso de fipronil e sulfluramida no controle de formigas cortadeiras.

Nesse sentido, o presente trabalho procurou avaliar a utilização de um ingrediente ativo com ação sistêmica, preparado junto ao condicionador de solo e aplicado diretamente às mudas no campo, para controle das formigas cortadeiras.

\section{MATERIAIS E MÉTODOS}

O experimento foi instalado em uma das áreas da Empresa Celulose Irani S.A., mais precisamente na Fazenda Campina da Alegria, no município de Vargem Bonita, SC, local denominado pela empresa de DNER, talhão CAM0180, com 6,32 ha de área, vizinha a uma área de APP e a um plantio de Eucalyptus sp. Não foi realizado controle prévio de formiga cortadeira (Figura 1).

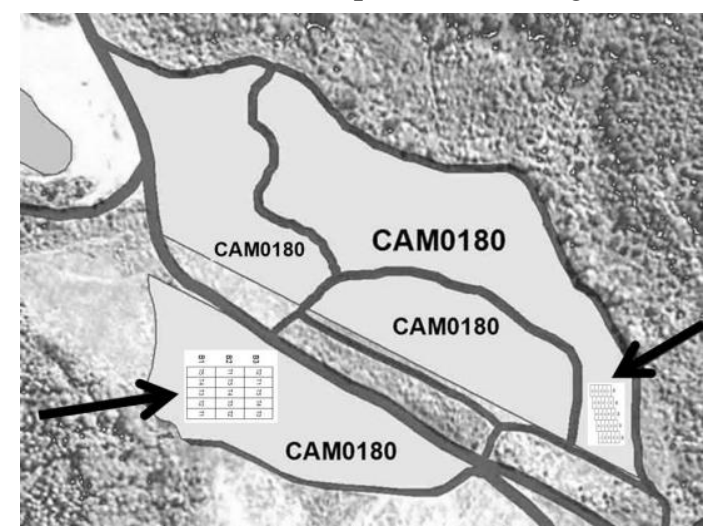

Figura 1. Indicação das áreas em que o experimento foi instalado no talhão CAM0180.

Figure 1. Indication of areas of installation of the experiment - CAM0180. 
Foi utilizado um delineamento experimental do tipo blocos ao acaso, com 5 tratamentos e 8 repetições, que foram instalados em 2 áreas do mesmo talhão (Figura 2). Cada tratamento foi constituído por 40 plantas, distribuídas em 4 linhas de plantio, com 10 plantas por linha, totalizando 1.600 mudas em todo o experimento.

Foram preparadas duas soluções com o ingrediente ativo imidacloprid. Na primeira, foram diluídos 20 gramas do ingrediente ativo em 100 litros de água $(0,2 \mathrm{~g} / \mathrm{litro})$ e na segunda foram diluídos 40 gramas em 100 litros de água $(0,4 \mathrm{~g} /$ litro). Após o preparo das caldas, foram então adicionados 200 gramas de gel condicionador de solo em cada uma delas.

Os tratamentos foram os seguintes: I - $50 \mathrm{ml}$ de gel na concentração $0,2 \mathrm{~g}$; II $-100 \mathrm{ml}$ de gel na concentração $0,2 \mathrm{~g}$; III - $50 \mathrm{ml}$ de gel na concentração $0,4 \mathrm{~g}$; IV - $100 \mathrm{ml}$ de gel na concentração $0,4 \mathrm{~g}$; e $\mathrm{V}$ - testemunha (sem aplicação do gel). O gel foi aplicado diretamente sobre as mudas após o plantio - I, II, III e IV (Tabela 1 e Figura 2). Para o preparo dos diferentes tratamentos, foram utilizados EPIs.

Tabela 1. Tratamentos utilizados.

Table 1. Treatments.

\begin{tabular}{lcc}
\hline Tratamentos & Concentração de i.a. & Volume de solução aplicado por muda \\
\hline I & $0,2 \mathrm{~g}$ & $50 \mathrm{ml}$ \\
II & $0,2 \mathrm{~g}$ & $100 \mathrm{ml}$ \\
III & $0,4 \mathrm{~g}$ & $50 \mathrm{ml}$ \\
IV & $0,4 \mathrm{~g}$ & $100 \mathrm{ml}$ \\
V (testemunha) & - & - \\
\hline
\end{tabular}

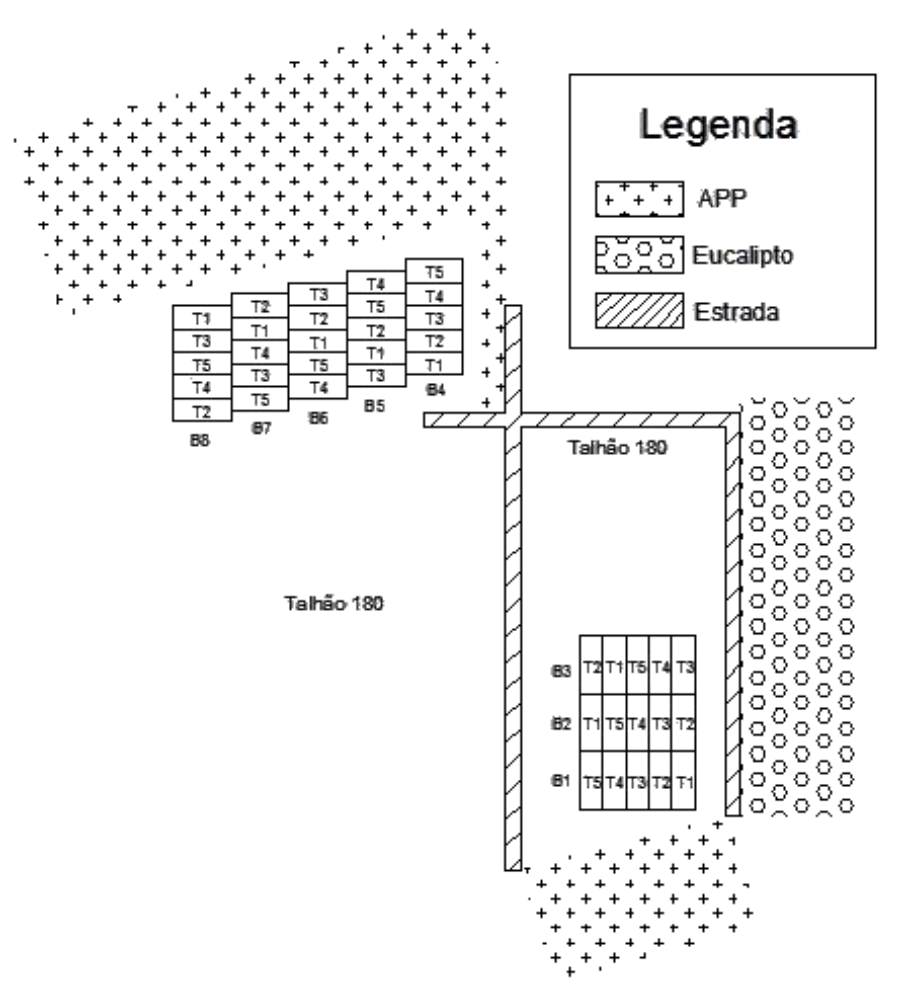

Figura 2. Croqui do experimento. Autora: Taise C. P. Arenhardt.

Figure 2. Experiment sketch. Author: Taise C. P. Arenhardt.

Foram realizadas 3 avaliações da porcentagem de dano causado por Acromyrmex spp., aos 25, 56 e 81 dias após a instalação do experimento. Foram considerados danos por formiga cortadeira desde mudas com corte mínimo de acícula até mudas apresentando retirada total das acículas e corte da gema apical (Figura 3). 


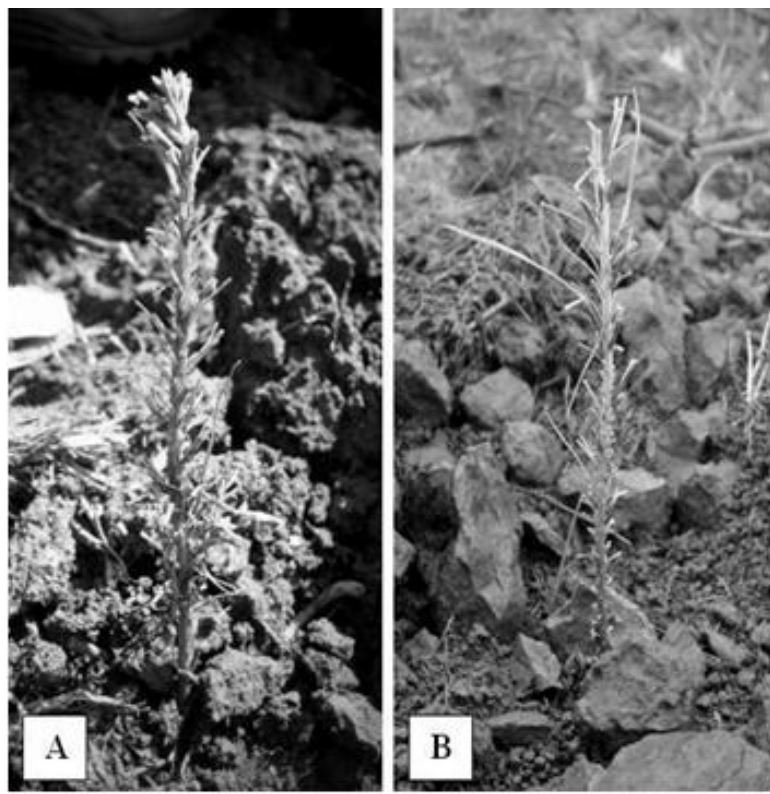

Figura 3. Diferentes intensidades de danos: (A) Corte de acículas; (B) Corte de acículas e gema apical. Figure 3. Different damages intensity: (A) cut of aciculae; (B) cut of aciculae and apical buds.

\section{RESULTADOS E DISCUSSÕES}

$\mathrm{Na} 1^{\text {a }}$ avaliação de dano, 25 dias após a instalação do experimento, foram identificadas 11 mudas danificadas por formiga cortadeira $(0,69 \%)$. Na $2^{\mathrm{a}}$ avaliação, após 56 dias, 29 mudas $(1,81 \%)$ foram atacadas, e na última avaliação, após 81 dias da instalação do experimento, 65 mudas $(4,06 \%)$ apresentaram dano (Figura 4).

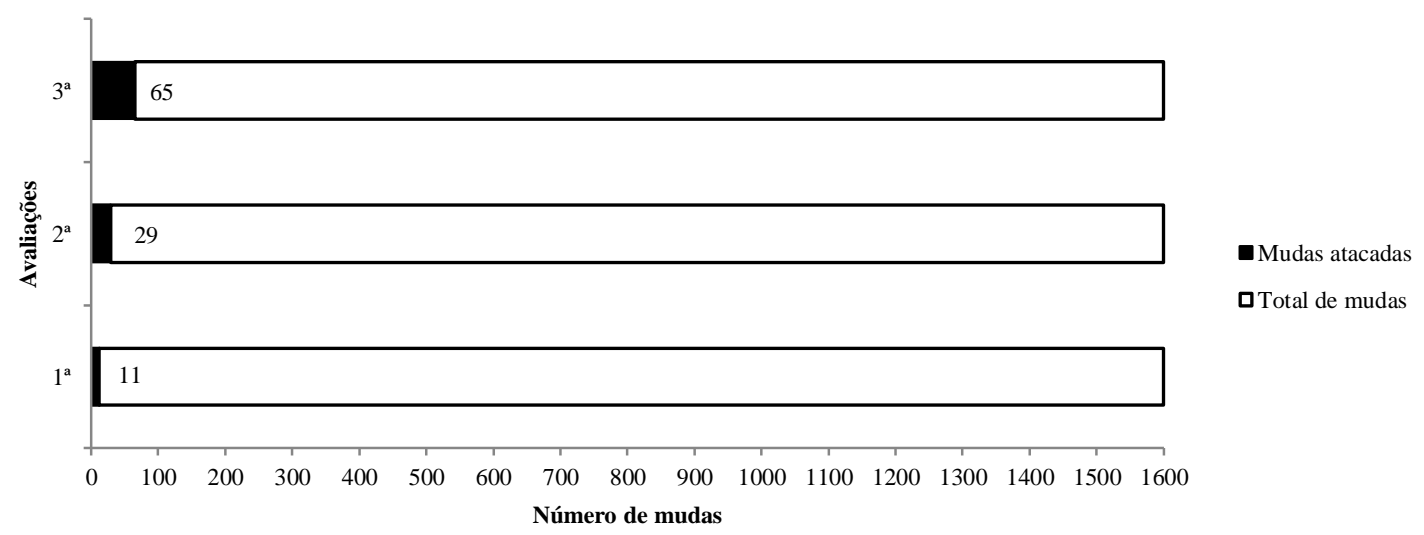

Figura 4. Valor acumulado de mudas atacadas por avaliação.

Figure 4. Accumulated value of seedlings attacked per evaluation.

Na primeira avaliação, os tratamentos I e IV não apresentaram mudas atacadas por formigas cortadeiras; o tratamento II apresentou o maior número de mudas atacadas, 6 mudas $(1,88 \%)$; o tratamento III apresentou 2 mudas com dano (0,63\%); e o tratamento V (testemunha) apresentou 3 mudas atacadas $(0,94 \%)$.

A segunda avaliação apresentou um aumento no número de mudas atacadas. O tratamento I apresentou 2 mudas atacadas $(0,63 \%)$ e no tratamento II foram 5 mudas atacadas $(1,56 \%)$, portanto 7 novas ocorrências em relação à $1^{\mathrm{a}}$ avaliação. No tratamento III, o número de mudas atacadas foi 
acrescido de $4(1,25 \%)$ em relação à $1^{\text {a }}$ avaliação, e no tratamento IV o número de mudas atacadas também foi acrescido de $4(1,25 \%)$. Já no tratamento $\mathrm{V}$, o número de novas mudas atacadas foi de 3 $(0,94 \%)$, mesmo número em relação à $1^{\mathrm{a}}$ avaliação.

Ao final das avaliações (81 dias), o tratamento I apresentou 11 novas mudas atacadas $(3,44 \%)$ e o tratamento II teve apenas 1 nova muda atacada $(0,31 \%)$. O tratamento III apresentou o maior número de novas mudas com danos - no total foram 18 mudas atacadas (5,63\%), 14 a mais em relação à avaliação anterior. O tratamento IV teve 2 novas mudas atacadas, o que representa $0,63 \%$, e o tratamento $\mathrm{V}$ (testemunha) apresentou 4 novas mudas atacadas $(1,25 \%)$. No total, foram 36 mudas com dano ao final da $3^{\mathrm{a}}$ avaliação, 18 mudas a mais em relação à $2^{\mathrm{a}}$ avaliação. Ao final da $3^{\mathrm{a}}$ avaliação, a porcentagem de dano no experimento foi de 4,06\% $(n=65)$ (Figura 5).

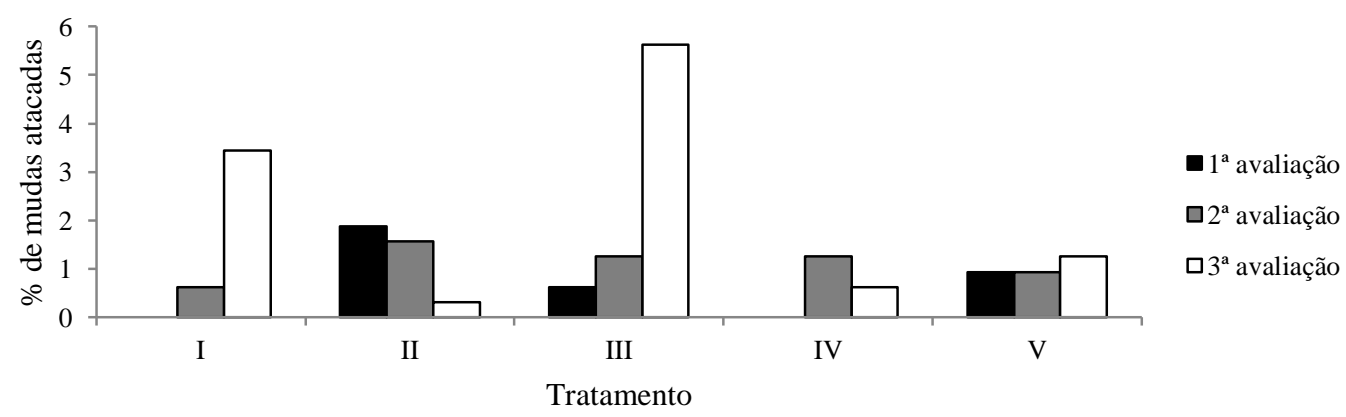

Figura 5. Porcentagem de mudas danificadas por formiga cortadeira em cada tratamento, na $1^{\mathrm{a}}, 2^{\mathrm{a}}$ e $3^{\mathrm{a}}$ avaliação.

Figure 5. Percentage of seedlings damaged by leaf cutting ant in each treatment in the 1st, 2nd and 3rd evaluation.

A análise estatística dos tratamentos demonstrou, através do teste de normalidade, que o padrão de distribuição foi não paramétrico.

Os tratamentos não apresentaram diferença estatística entre si (Figura 2). A avaliação dos dados sugere que os tratamentos I e III, onde foram utilizados $50 \mathrm{ml}$ de solução, propiciou um maior ataque das formigas na $3^{\text {a }}$ avaliação, uma vez que a quantidade de gel pode não ter sido suficiente para maior proteção das mudas. Recomenda-se a utilização da maior quantidade de solução $(100 \mathrm{ml}) \mathrm{com}$ a menor concentração de ingrediente ativo $(0,2 \mathrm{~g})$, visando menores custos e efeitos prejudiciais ao meio ambiente.

Tabela 2. Análise de Kruskal-Wallis para os tratamentos ao nível de 1\% de probabilidade.

Table 2. Kruskal-Wallis analysis to the treatments $-1 \%$ of probability.

\begin{tabular}{lcccc}
\hline Tratamento & Repetições & Média & Soma dos pontos & Classificação \\
\hline I & 8 & 1,625 & 175,50 & a \\
II & 8 & 0,75 & 135,00 & a \\
III & 8 & 3,00 & 199,00 & a \\
IV & 8 & 1,50 & 154,50 & a \\
V & 8 & 1,25 & 156,00 & a \\
\hline
\end{tabular}

A intensidade total de dano ao final do experimento (81 dias) foi de 4,06\%, resultado promissor para o controle de Acromyrmex spp., uma vez que boa parte das empresas do setor florestal no sul do Brasil aceitam 5\% como limite máximo de perdas em implantação de plantios. Segundo Buratto et al. (2012), com a utilização de iscas formicidas os danos totais médios ocasionados por formigas cortadeiras em mudas de P. taeda no município de Anita Garibaldi, SC, foi no máximo de 5,38\% no $63^{\circ}$ dia de avaliação. Importante ressaltar que a forma de controle testada, diferentemente do controle com iscas formicidas, não elimina a colônia, permitindo que elas permaneçam ativas, uma vez que, de acordo com Nickele et al. (2012), a espécie Acromyrmex crassispinus causa perdas em indivíduos de P. taeda somente nos primeiros meses de idade do plantio, com maior concentração nos primeiros 30 dias em

FLORESTA, Curitiba, PR, v. 45, n. 1, p. 41 - 48, jan. / mar. 2015.

Vitorino, M. D. et al.

ISSN eletrônico 1982-4688 / ISSN impresso 0015-3826

45

DOI: $10.5380 /$ rf.v45i1.31912 
áreas do planalto norte do estado de Santa Catarina. Não foram previamente contados os formigueiros presentes na área, mas o experimento só foi instalado após a confirmação de que havia formiga cortadeira no entorno.

Os tratamentos II e IV não apresentaram picos de ataque e seguiram um padrão de ataque muito próximo ao observado na testemunha. Já os tratamentos I e III, embora estatisticamente não tenham apresentado diferenças significativas em relação aos demais tratamentos, apresentaram pico de ataque, principalmente o tratamento III (Figura 5), o que sugere que a quantidade de solução aplicada nas mudas afetou os níveis de ataque.

Em relação aos blocos, B4 e B8 apresentaram os maiores níveis de dano, com 22 mudas atacadas $(11 \%)$ em cada um (Figura 6). Isso pode ser explicado pela maior influência que tiveram da APP, uma vez que, de acordo com Klitzke et al. (2011) e Arenhardt et al. (2012), a maior concentração de colônias de formigas cortadeiras ocorre próxima às APPs. Os demais blocos apresentaram níveis de dano entre 0,5 e 3,5\%, inclusive o bloco B1, que também era influenciado pela APP, porém de pequenas dimensões $\left(200 \mathrm{~m}^{2}\right)$, não oferecendo condições de suporte para a presença das formigas cortadeiras.

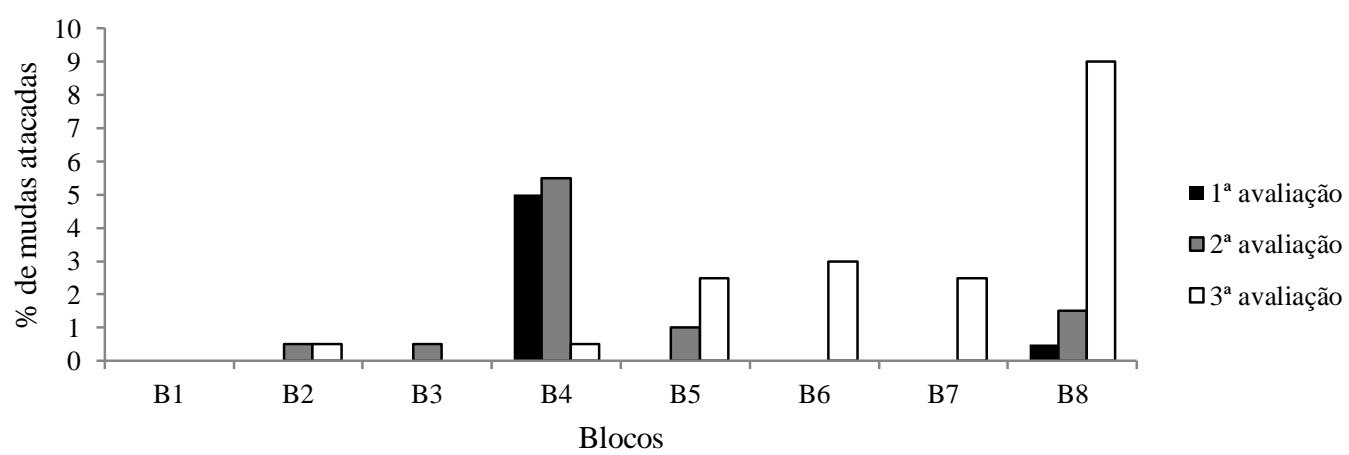

Figura 6. Porcentagem de mudas danificadas por formiga cortadeira em cada bloco, na $1^{\mathrm{a}}, 2^{\mathrm{a}}$ e $3^{\mathrm{a}}$ avaliações.

Figure 6. Percentage of seedlings damaged by leaf cutting ant in each block, in the 1st, 2nd and 3rd evaluations.

Para os blocos, o teste de normalidade resultou em dados não paramétricos e, na análise de Kruskal-Wallis, demonstrou haver diferença estatística ao nível de 1\% de probabilidade (Tabela 3).

Tabela 3. Análise de Kruskal-Wallis para os blocos ao nível de $1 \%$ de probabilidade.

Table 3. Kruskal-Wallis analysis to the blocks- $1 \%$ of probability.

\begin{tabular}{lcccc}
\hline Blocos & Repetições & Média & Soma dos pontos & Classificação \\
\hline B1 & 5 & 0,0 & 42,50 & $\mathrm{f}$ \\
B2 & 5 & 0,4 & 70,50 & $\mathrm{~d}$ \\
B3 & 5 & 0,2 & 56,50 & $\mathrm{e}$ \\
B4 & 5 & 4,4 & 173,50 & $\mathrm{~A}$ \\
B5 & 5 & 1,4 & 128,50 & $\mathrm{C}$ \\
B6 & 5 & 1,2 & 120,50 & $\mathrm{C}$ \\
B7 & 5 & 1,0 & 83,50 & $\mathrm{D}$ \\
B8 & 5 & 4,4 & 144,50 & $\mathrm{~B}$ \\
\hline
\end{tabular}

Os blocos B4 e B8, por sofrerem influência da APP, provavelmente serviram de barreira de proteção para os demais blocos dentro do experimento, pois, eliminando o número de ataques desses dois blocos, a porcentagem final de ataque cai para $1,31 \%$. Essa influência pode estar relacionada com a ação de semioquímicos, de acordo com Ridley et al. (1996). Semioquímicos do fungo transferidos através da trofalaxia determinam a seleção de plantas pelas forrageadoras. Dessa forma, as formigas cortadeiras conseguem detectar materiais vegetais que contenham elementos químicos indesejáveis ao fungo. Em 
função disso, uma formiga que entrar em contato com alguma substância incomum e tóxica às demais formigas e à colônia, será separada rapidamente pelas operárias, pois poderia provocar mudanças comportamentais na colônia (MARINHO et al., 2006).

O bloco B7 apresentou danos apenas na $3^{\mathrm{a}}$ avaliação nos tratamentos I e III, o que confirma a conclusão de que a quantidade de solução (quantidade de gel e ingrediente ativo) aplicada nas mudas afetou a porcentagem de dano nas mesmas, uma vez que as que mais apresentaram dano foram tratadas com $50 \mathrm{ml}$ de solução.

Os blocos B5 e B6 são estatisticamente iguais, por se localizarem um ao lado do outro no experimento e por estarem entre outros blocos, não sofrendo influência das bordas do talhão.

Os blocos B1, B2 e B3 apresentaram baixos níveis de dano, representando apenas 0,19\% do total de mudas avaliadas. Esses blocos se localizavam no mesmo talhão, porém em uma área de 1,63 ha ( $25,8 \%$ da área total do talhão), separada dos demais blocos por uma estrada e margeada por plantios de Eucalyptus e uma área de APP de $200 \mathrm{~m}^{2}$, que não era grande o suficiente para causar influência nos tratamentos/blocos (Figura 1).

Extrapolando a forma de controle apresentada neste estudo para a área de 1 ha, seriam necessários $400 \mathrm{~g}$ de ingrediente ativo em uma calda de 200 litros.

\section{CONCLUSÕES}

- A calda com $100 \mathrm{ml}$ de gel e 0,2 gramas do ingrediente ativo imidacloprid por muda apresentou melhor controle.

- A presença da APP próxima aos plantios influenciou o ataque de formigas cortadeiras.

- A intensidade de dano após três avaliações (81 dias) foi de $4,06 \%$ de perdas.

\section{AGRADECIMENTOS}

À empresa Celulose Irani S/A, pelo apoio logístico e disponibilização do talhão para estudo, e à Fundação de Amparo à Pesquisa e Inovação do Estado de Santa Catarina (FAPESC), pelo financiamento concedido.

\section{REFERÊNCIAS}

ANJOS, N.; MOREIRA, D. D.; DELlA LUCIA, T. M. C. Manejo integrado de formigas em reflorestamentos. In: DELLA LUCIA, T. M. C. (Ed.). As formigas cortadeiras. Viçosa, 1993. p. 212 - 241.

ANTUNES, E. C.; DELLA LUCIA, T. M. C. Consumo foliar em Eucalyptus urophylla por Acromyrmex laticeps nicrosetosus Forel (Hymenoptera - Formicidae). Ciência e Agrotecnologia, Lavras, v. 23, p. 208 - 211, 1999.

ARENHARDT, T. C. P.; DUTRA, R. N.; GONÇALVES, A. H. M.; VITORINO, M. D. Redução na quantidade de iscas formicidas através do método de amostragem de colônias de formiga cortadeira. In: MOSTRA INTEGRADA DE ENSINO, PESQUISA E EXTENSÃO - MIPE, 6., 2012, Blumenau. Anais... Blumenau, p. 57, 2012.

BURATTO, D. A.; CARDOSO, J. T.; ROLIM, F. A.; REIS FILHO, W. Avaliação dos danos causados por formigas cortadeiras do gênero Acromyrmex (Hymenoptera) aos plantios de Pinus taeda no planalto sul catarinense. Revista Floresta, Curitiba, v. 42, n. 4, p. 683 - 690, 2012.

CANTARELLI, E. B.; COSTA, E. C.; PEZZUTTI, R.; OLIBEIRA, L. S. Quantificação das perdas no desenvolvimento de Pinus taeda após o ataque de formigas cortadeiras. Ciência Florestal, Santa Maria, v. 18 , n. 1 , p. $39-45,2008$.

KLITZKE, A.; VITORINO, M. D.; RANUCI, L. E. C.; HOLLER, K. R.; PRADE, P. Aplicação da amostragem de formigueiros para a diminuição do uso de iscas formicidas em povoamentos de Pinus taeda no meio-oeste catarinense. Dynamis Revista Técnico Científica, v. 16, n. 2, p. 4- 5, 2011. 
MARINHO, C. G. S.; DELlA LUCIA, T. M. C.; PICANÇO, M. C. Fatores que dificultam o controle de formigas cortadeiras. Bahia Agrícola, Salvador, v. 7, n. 2, p. 18 - 21, 2006.

MENDES FILHO, J. M. de A. Técnica de combate às formigas. Série Técnica - IPEF, Piracicaba, v. 2, n. 7, 1981. 19 p.

NICKELE, M. A.; REIS FILHO, W.; OlIVEIRA, E. B. de; IEDE, E. T. CALDATO, N.; STRAPASSON, P. Attack of leaf-cutting ants in initial pine plantations and growth of plants artificially defoliated. Pesquisa Agropecuária Brasileira, Brasília, v. 47, p. 892 - 899, 2012.

NICKELE, M. A. Distribuição espacial, danos e planos de amostragem de Acromyrmex crassispinus (Forel, 1909) (Hymenoptera: Formicidae) em plantios de Pinus taeda L. (Pinaceae). $111 \mathrm{f}$. Dissertação (Mestrado em Entomologia) - Universidade Federal do Paraná, Curitiba, 2008.

REZENDE, J. P.; PEREIRA, A. R.; OLIVEIRA, A. D. Espaçamento ótimo para a produção de madeira. Revista Árvore, v. 7, n. 1, p. 30 - 43, 1983.

RIBEIRO, G. T.; MENDONÇA, M. C. Controle de formigas cortadeiras. In: SEMINÁRIO SOBRE SILVICULTURA EM FLORESTAS PLANTADAS, 1., 2004, Vitória. Anais do... Viçosa, 2004, p. 40.

RIDLEY, P.; HOWSE, P. E.; JACKSON, C. W. Control of the behaviour of leaf-cutting ants by their 'simbiotic' fungus. Experientia, v. 52, n. 6, p. 631 - 635, 1996.

VILELA, E. F. Status of leaf-cutting ant control in forest plantation in Brazil, In: LOFGREEN, C. S. VAN DER MEER, R. K. (Eds.). Fire ants leaf-cutting ants: biology and management. Bouder: Westview press. 1986, p. 399 - 408.

VITORINO, M. D.; BEAL, L. C.; DUTRA, R. N.; PRADE, P.; ARENHARDT, T. C. P. Diminuição do uso de iscas formicidas em povoamentos de Pinus taeda no meio oeste catarinense através da amostragem de colônias. In: XXIV CONGRESSO BRASILEIRO DE ENTOMOLOGIA, 24., 2012, Curitiba. Anais... Curitiba, 2012, p. 757. 